\title{
Cloverleaf skull-multiple congenital anomalies syndrome
}

INSERM

\section{Source}

INSERM. (1999). Orphanet: an online rare disease and orphan drug data base. Cloverleaf skull-multiple congenital anomalies syndrome. ORPHA:93267

This newly described syndrome is characterized by cloverleaf skull, limb anomalies, facial dysmorphism and multiple congenital anomalies. 